\title{
Effect of antenatal exercise on mode of delivery in gestational diabetic females: a single-blind randomized controlled trial
}

\author{
DOI: https://doi.org/10.5114/pq.2019.84270
}

\author{
Eman Awad', Hamada Ahmed ${ }^{2}$, Amal Yousef ${ }^{3}$, Ibtissam M. Saab ${ }^{4}$ \\ ${ }^{1}$ Physical Therapy Department for Woman and Child Health, Faculty of Physical Therapy, Beni-Suef University, \\ Beni-Suef, Egypt \\ ${ }^{2}$ Department of Biomechanics, Faculty of Physical Therapy, Cairo University, Cairo, Egypt \\ ${ }^{3}$ Department of Physical Therapy for Gynaecology and Obstetrics, Faculty of Physical Therapy, Cairo University, \\ Cairo, Egypt \\ ${ }^{4}$ Physical Therapy Department, Faculty of Health Sciences, Beirut Arab University, Beirut, Lebanon
}

Abstract

Introduction. Gestational diabetes mellitus (GDM) is common in pregnancy. Maternal consequences might include an increased rate of caesarean delivery. This study was conducted to determine the effect of an exercise program on the mode of delivery in gestational diabetic females.

Methods. A prospective, randomized, single-blind, pre-post-test, controlled trial was performed. Overall, 60 pregnant females with GDM were included, at their 20-24 weeks of gestation, aged 25-35 years, with body mass index not exceeding $40 \mathrm{~kg} / \mathrm{m}^{2}$. The participants were randomly assigned into 2 equal groups: group A, undergoing an exercise program with a moderately restricted diet and insulin therapy, and group B (control group), receiving solely the same diet protocol with insulin therapy.

Results. The chi-square test revealed significant differences between the groups in the mode of delivery $(p<0.05)$, with a significant decrease in caesarean deliveries in group A. Group A showed a statistically significant difference in neonates' Apgar scores at the $1^{\text {st }}$ and $5^{\text {th }}$ minute of life $(p<0.05)$ compared with the participants in group B.

Conclusions. It can be concluded that antenatal exercises can be considered effective in decreasing labour complications and shifting the mode of delivery towards normal, complication-free delivery in females with GDM and their offspring.

Key words: antenatal exercises, gestational diabetes, neonates, pregnancy, Apgar score, physical activity

\section{Introduction}

Diabetes mellitus is one of the most common medical complications in pregnancy. Gestational diabetes mellitus (GDM) is defined as carbohydrate intolerance of variable severity with onset or first recognition during pregnancy [1, 2]. GDM affects 3-9\% of pregnancies, depending on the population studied. It is especially common during the last 3 months of pregnancy [3]. It is associated with adverse outcomes not only for the mother, but also for the child, whether as a foetus, a neonate, a child, or an adult. Maternal consequences include an increased rate of operative and caesarean delivery, hypertensive disorders during pregnancy, and future risk for type 2 diabetes mellitus, as well as other aspects of metabolic syndrome, such as obesity, cardiovascular morbidities, and recurrent GDM [4, 5]. Also, there are maternal implications secondary to a delivery of a macrosomic foetus, such as an increased rate of caesarean delivery, postpartum haemorrhage, birth trauma, and shoulder dystocia [6-8]. It is widely assumed that caesarean delivery results in higher rates of maternal morbidity and mortality compared with vaginal delivery. Additional evidence exists for a 2-4-fold greater risk of maternal death in women who delivered by caesarean delivery compared with vaginal delivery [9]. In the foetus or neonate, the disorder is associated with higher rates of perinatal mortality, macrosomia, birth trauma, hyperbilirubinemia, and neonatal hypoglycaemia [10, 11]. In general, screening and diagnostic tests are performed between 24 and 28 weeks because it is at this point in gestation that the diabetogenic effect of pregnancy is usually manifested [12]. GDM is treated with a diabetic diet, exercise, and possibly insulin injections [3]. Researches have shown that the most physically active women have the lowest prevalence of GDM [13]. The American Diabetes Association and the International Federation of Gynecology and Obstetrics both recommended that lifestyle management including physical activity should be the first choice in the treatment of GDM [14]. Some authors concluded a 1/3 reduction in comorbidities associated with GDM, particularly the risk of acute or elective caesarean delivery [15]. Others reported a reduction in complications associated with pregnancy in GDM women [16]. Despite these conclusions and recommendations, relatively few are studies examining the effect of regular exercise with dietary intervention in the management of GDM and/or in preventing complications associated with GDM. Therefore, the aim of the current study was to determine the effect of a selected exercise program on the delivery mode in GDM women.

\section{Subjects and methods}

\section{Design}

The study was designed as a controlled trial that compared 2 groups: group A included participants who performed the

Correspondence address: Hamada Ahmed, Department of Biomechanics, Faculty of Physical Therapy, Cairo University, 7 Ahmed Elziat Street, Bean Elsariat, El Dokki, Giza, Cairo, Egypt, e-mail: Hamada.Ahmed@pt.cu.edu.eg

Received: 31.01 .2019

Accepted: 25.02.2019

Citation: Awad E, Ahmed H, Yousef A, Saab IM. Effect of antenatal exercise on mode of delivery in gestational diabetic females: a single-blind randomized controlled trial. Physiother Quart. 2019;27(2):1-5; doi: https://doi.org/10.5114/pq.2019.84270. 
exercise program starting from 24 weeks of gestation till the date of delivery, with a moderately restricted diet, typically with 2000-2500 kcal, and insulin therapy [17], while group B involved control participants, who received solely the same diet as group A with insulin therapy. Anonymity was assured through coding of all data. The women were randomly assigned into 2 groups by a blinded and an independent research assistant, who opened sealed envelopes that contained a computer-generated randomization card. No subjects dropped out of the study after randomization.

\section{Sampling}

During the 2-year recruitment period from May 2015 to May 2017, 60 pregnant females diagnosed with GDM at their 20-24 weeks of gestation were recruited from the Outpatient Clinic of Bab El-Sharia Hospital, Cairo, Egypt. Their age ranged between 25 and 35 years and their body mass index (BMI) did not exceed $40 \mathrm{~kg} / \mathrm{m}^{2}$. Females diagnosed with vascular complications, unstable diabetes mellitus, peripheral neuropathy, autonomic dysfunction, nephropathy or retinopathy, twins, placenta praevia, foetal anomalies, intrauterine growth retardation, as well as with a previous history of preterm labour, repeated abortions, antepartum haemorrhage, or preeclampsia were excluded from the study.

\section{Procedure}

The participants were randomly assigned into 2 groups equal in number; each group included 30 pregnant women on the basis of a block-style randomization scheme [18]. Additional screening for specific inclusion and exclusion criteria and demographic data was taken, including age and BMI. A full assessment of history was performed for each patient in both groups prior to the start of the study in accordance with the items of the data recording sheet. The 3-hour oral glucose tolerance test was conducted for both groups before starting the treatment and at 37 weeks of gestation.

All exercise sessions were held by the same physiotherapist. The exercise program parameters were in concordance with the American College of Obstetricians and Gynecologists (ACOG) guidelines for exercise during pregnancy for sedentary women and with the FITT principle (frequency, intensity, time, and type). The exercise undertaken was characterized by a frequency of minimum 3 times per week, intensity set at moderately hard perceived exertion, time of 60 minutes per day, and type of low impact. These exercise guidelines were utilized for both aerobic and strength exercises for 12 weeks [19]. Moderate-intensity physical activities referred to activities that required moderate physical effort, which made the pregnant women's breath slightly harder and their heart beat a little faster than normal.

Group A participants performed the exercise 3-4 times per week, starting the active phase of aerobic training with 15 minutes at a target heart rate intensity and increasing the time gradually to a maximum of 30 minutes per exercise session. Each aerobic activity was preceded by a 10-15-minute warm-up and followed by a 10-15-minute cool-down. The exercise intensity was monitored with the use of target heart rate zones, the Borg scale (rating of perceived exertion), or the 'talk test' [20]. Heart rate zones provided in the ACOG guideline corresponded to moderate-intensity exercise (i.e. $60-80 \%$ of maximal aerobic capacity, $\mathrm{VO}_{2} \mathrm{max}$ ). The type of aerobic exercise was walking on treadmill to train large muscle groups. Next, circuit resistance training (CRT) exercises were performed with 2 circuits, each of 10 repetitions (with a 2-minute rest between each circuit), with the use of the green colour band (green colour: $1.36 \mathrm{~kg}$, during the first 4 weeks of the training program) and then the blue colour band (blue colour: $1.8 \mathrm{~kg}$, until the end of 37 weeks gestation), with elastic resistance performed at $100 \%$ elongation for 15 minutes. The main muscle groups exercised were chest, deltoid, quadriceps, and calf muscles. CRT was performed from a sitting position; one end of the band was fixed beneath the feet, held for 5 seconds, and then released. The exercises included chest push, shoulder flexion, shoulder abduction, knee extension, and ankle planter flexion exercise [21]. The subjects were taught to monitor their own heart rate during exercise to ensure that it did not rise above 140 beats/min. The pregnant women were advised to immediately stop exercising if they exhibited symptoms such as dizziness, dyspnoea, amniotic fluid leaking, or vaginal bleeding [22]. Moreover, group A participants wore a loose-fitting clothing and kept hydrated while exercising in an environment with appropriate temperature and humidity [23]. All exercise sessions were recorded in a log book.

\section{Outcome measures}

\section{Mode of delivery}

It involved normal vaginal delivery, instrumental vaginal delivery that required the use of special devices such as forceps or a vacuum extractor to deliver the foetus vaginally, and caesarean section.

\section{Apgar score}

The Apgar score was applied to assess the neonates' condition during the critical $1^{\text {st }}$ and $5^{\text {th }}$ minutes of life in both groups, A and B. The Apgar score was determined by evaluating the newborn on 5 simple criteria, referred to as appearance, pulse, grimace, activity, and respiration to aid mnemonic learning. The resulting Apgar score ranged from 0 to 10 . Scores below 3 were generally considered critically low, 4-6 as fairly low, and higher than 7 as generally normal [24].

\section{Data analysis}

All statistical procedures were performed with the Statistical Package for the Social Sciences (SPSS) software, version 23 for Windows. The test involved two independent variables. The first one was the tested group, a between-subject factor which had 2 levels (group A receiving exercise, diet, and insulin, while group B only receiving diet and insulin therapy). The second variable was the neonates' Apgar score (measuring periods), a within-subject factor which had 2 levels (after 1 minute and after 5 minutes). Additionally, the test involved one dependent variable: the neonates' Apgar score between groups. Preliminary assumption checking revealed that data were normally distributed, as assessed by a normal Q-Q plot. The examination of studentized residuals for values greater than \pm 3 proved that there were no outliers. Homogeneity of variances $(p>0.05)$ and covariances $(p>0.05)$ was observed in Levene's test of homogeneity of variances and Box's $M$ test, respectively. All these findings allowed the researchers to conduct parametric analysis. So, $2 \times 2$ mixed design ANOVA was used to compare the tested variables of interest in different tested groups and measuring periods. The alpha level was set at 0.05 . 


\section{Ethical approval}

The research related to human use has been complied with all the relevant national regulations and institutional policies, has followed the tenets of the Declaration of Helsinki, and has been approved by the ethical committee at the Physical Therapy Department of Bab El-Sharia University Hospital and by the institutional review board at the Faculty of Physical Therapy, Cairo University.

\section{Informed consent}

Informed consent has been obtained from all individuals included in this study.

\section{Results}

There were no statistically significant differences $(p>0.05)$ between the subjects in both groups concerning age, BMI, or gestational age. However, the chi-square test revealed significant differences between the groups in the mode of delivery distribution $(p<0.05)$ (Table 1).

The statistical analysis with mixed design ANOVA analysed 60 patients assigned into 2 equal groups. It revealed a significant within-subject effect $(F=306.82, p=0.0001)$ and between-subject effect $(F=50.129, p=0.0001)$. However, there was no significant treatment ${ }^{\star}$ time effect $(F=0.58, p=$ 0.449 ). Table 2 presents the descriptive statistics (mean \pm SD) and multiple pairwise comparison tests (post-hoc tests) of the detective variable. In the same context regarding the withinsubject effect, the multiple pairwise comparison tests revealed that there was a significant increase $(p<0.05)$ in the Apgar score after 5 minutes compared with that after 1 minute in the neonates of both groups. As for the between-subject effects, multiple pairwise comparisons showed a significant increase in favour of group $A$ as compared with group $B$ at 1 minute and 5 minutes $(p<0.05)$.

\section{Discussion}

A significant number of pregnant women are affected by GDM every year. Its growing prevalence and established relationship to numerous complications and disorders during and after pregnancy demand identification of factors that can prevent the incidence and influence its course. Therefore, this study was conducted to determine the effect of a specialized antenatal exercise program on the mode of delivery and neonatal condition among 60 females suffering from GDM.

The results revealed a statistically significant difference in the mode of delivery distribution, with a significant decrease in the number of caesarean deliveries in the study group compared with the control group. Also, there was a statistically significant increase in the neonates' Apgar scores at the critical $1^{\text {st }}$ and $5^{\text {th }}$ minutes after delivery, which favoured the neo-

Table 1. Demographic characteristics of both groups

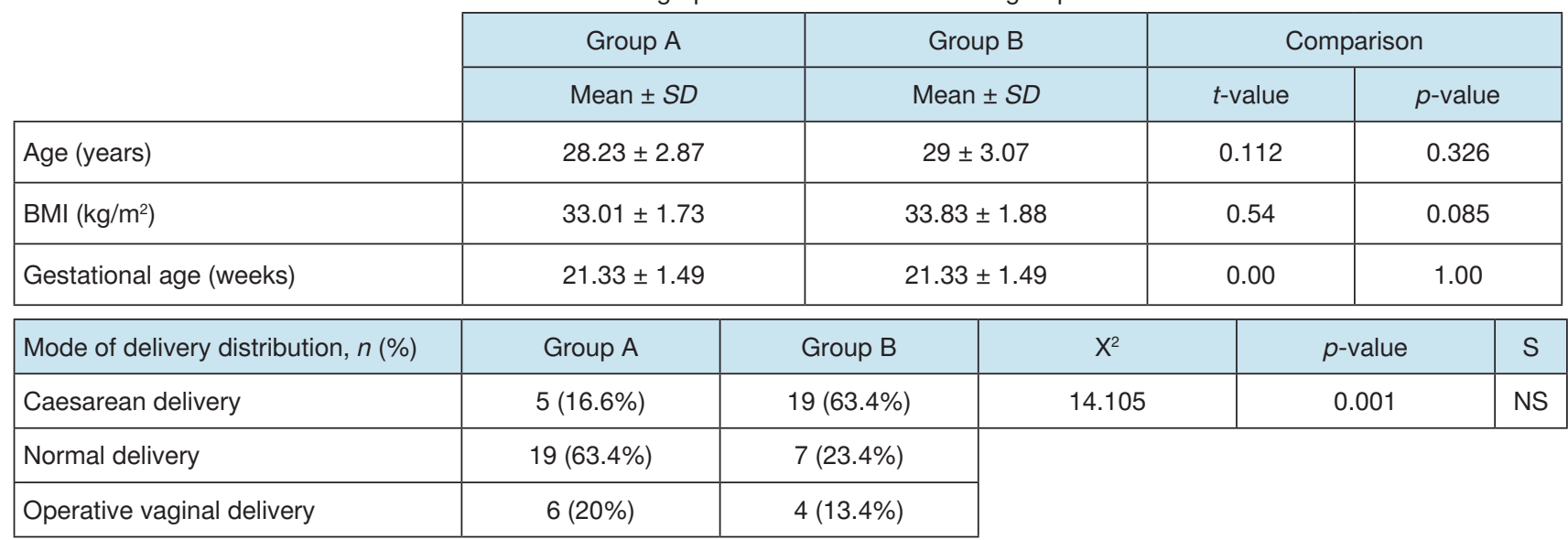

$S D$ - standard deviation, BMI - body mass index, $\mathrm{S}$ - significance, NS - non-significant

Table 2. Descriptive statistics and $2 \times 2$ mixed design ANOVA for Apgar score at different measuring periods in both groups

\begin{tabular}{|c|c|c|c|c|}
\hline Apgar score & $\begin{array}{c}\text { Group A } \\
(\text { Mean } \pm S D)\end{array}$ & Group B & Mean difference & $95 \% \mathrm{Cl}$ \\
\hline At $1^{\text {st }}$ minute & $7.23 \pm 0.43$ & $6.43 \pm 0.5$ & 0.8 & $(0.558-1.042)$ \\
\hline At $5^{\text {th }}$ minute & $8.7 \pm 0.46$ & $8.03 \pm 0.68$ & 0.66 & $(0.369-0.965)$ \\
\hline Mean difference & -1.46 & -1.6 & & \\
\hline $95 \% \mathrm{Cl}$ & (from -1.714 to -1.219 ) & (from -1.848 to -1.352 ) & & \\
\hline \multicolumn{5}{|c|}{ Multiple pairwise comparisons between Apgar score values after 1 minute and after 5 minutes } \\
\hline $1^{\text {st }}$ minute vs. $5^{\text {th }}$ minute & \multicolumn{2}{|c|}{ Group A } & \multicolumn{2}{|c|}{ Group B } \\
\hline$p$-value & \multicolumn{2}{|c|}{$0.0001^{*}$} & \multicolumn{2}{|c|}{$0.0001^{*}$} \\
\hline \multicolumn{5}{|c|}{ Multiple pairwise comparison tests (post-hoc tests) for the Apgar score between both groups at different measuring periods } \\
\hline & \multicolumn{2}{|c|}{ At $1^{\text {st }}$ minute } & \multicolumn{2}{|c|}{ At $5^{\text {th }}$ minute } \\
\hline Group A vs. group B & \multicolumn{2}{|c|}{$0.0001^{*}$} & \multicolumn{2}{|c|}{$0.0001^{*}$} \\
\hline
\end{tabular}

* significant at alpha level $<0.05$

SD - standard deviation, $95 \% \mathrm{Cl}-95 \%$ confidence interval 
nates of group A. These observations are in agreement with other reports stating that physical exercise is highly recommended to the broad population before and during pregnancy, and to women suffering from or at risk for GDM [25]. Women suffering from diabetes during pregnancy are more liable to develop hypertensive disorders and pre-eclampsia and have a higher risk of an induction of labour or caesarean section delivery [26]. Our findings are also supported by those who presented a $1 / 3$ reduction in the risk of undergoing acute or elective caesarean delivery among women who developed GDM and conformed to a regular moderate-intensity antenatal exercise program [15].

GDM is associated with an elevated risk for delivering a large-for-gestational-age or macrosomic infant. As a consequence of their size, the offspring of GDM mothers are more likely to suffer from significant birth trauma, such as shoulder dystocia, perinatal asphyxia, bone fractures, and nerve palsy. High foetal birth weight has also been claimed to impose additional risks of caesarean section and cephalopelvic disproportion [27].

Our findings are also supported by Lawani et al. [28], who reported a strong positive effect of adding aerobic and resistance antenatal exercises to the classical antenatal exercise programs on health behaviours and a vaginal lowrisk birth in females diagnosed with GDM. Antenatal exercise is a non-pharmacological childbirth preparation method. It is considered both a physical and a psychological training method in accordance with the natural mechanisms of childbirth. Antenatal exercises led to a lower rate of prolonged first stage of labour compared with women who received no training and also resulted in fewer delivery complications as poor muscle tone may cause incontinence, unusual pain during birth, prolonged first and second stages of labour. Regular exercise in the third trimester was associated with lower incidence of high foetal birth weight [29].

However, the results of our study are not in line with several others reporting no effects of exercise or lifestyle (combining diet and exercise) interventions during pregnancy on Apgar score or head circumference [30-32]. In a randomized controlled trial of 105 women, higher mean Apgar scores were observed at the $1^{\text {st }}$ minute, but not at the $5^{\text {th }}$ minute, among newborns of women allocated to training [30]. This was observed in a per-protocol analysis and not in an intention-to-treat analysis, and the Apgar score at the $5^{\text {th }}$ minute is considered a better sign of newborn wellbeing than that at the $1^{\text {st }}$ minute $[33,34]$.

The results of our study provide grounds for provision of advice as well as organizing exercise training groups for females diagnosed with GDM and emphasizing the importance of a healthy diet and being physically active to gain the recommended weight during pregnancy and after giving birth and to prevent pregnancy and delivery complications in resource-limited countries.

\section{Limitations}

Despite the design of the present study (a randomized controlled clinical trial), the small sample size recruited could be its potential limitation. On the basis of sample size estimation with the power of the study $1-B=80 \%$ to detect the effect size of $d=0.5$ with a significance level of $<0.05,50$ participants were needed for each group. At present, it is not possible to study the effect of each antenatal exercise on common risks associated with GDM.

\section{Conclusions}

It can be concluded that maintaining a physically active lifestyle throughout pregnancy protects patients against GDM complications. Many scientific and professional reports stress the importance of exercise as an adjunct therapy for GDM women. Antenatal exercises are proven to be effective in decreasing labour complications and shifting the mode of delivery towards normal, complication-free delivery in females diagnosed with GDM and their offspring.

\section{Disclosure statement}

No author has any financial interest or received any financial benefit from this research.

\section{Conflict of interest}

The authors state no conflict of interest.

\section{References}

1. Langer O. Management of gestational diabetes: pharmacologic treatment options and glycemic control. Endocrinol Metab Clin North Am. 2006;35(1):53-78; doi: 10.1016/j.ecl.2005.09.007.

2. Mclntyre HD. Discovery, knowledge, and action-diabetes in pregnancy across the translational spectrum: the 2016 Norbert Freinkel Award lecture. Diabetes Care. 2018;41(2):227-232; doi: 10.2337/dci17-0056.

3. Brite J, Shiroma EJ, Bowers K, Yeung E, Laughon SK, Grewal JG, et al. Height and the risk of gestational diabetes: variations by race/ethnicity. Diabet Med. 2014;31(3): 332-340; doi: 10.1111/dme.12355.

4. Yogev $Y$, Xenakis EM, Langer $O$. The association between preeclampsia and the severity of gestational diabetes: the impact of glycemic control. Am J Obstet Gynecol. 2004;191(5):1655-1660; doi: 10.1016/j.ajog.2004. 03.074 .

5. Kim C, Newton KM, Knopp RH. Gestational diabetes and the incidence of type 2 diabetes: a systematic review. Diabetes Care. 2002;25(10):1862-1868; doi: 10.2337/diacare.25.10.1862.

6. Sermer M, Naylor CD, Gare DJ, Kenshole AB, Ritchie JW, Farine D, et al. Impact of increasing carbohydrate intolerance on maternal-fetal outcomes in 3637 women without gestational diabetes. The Toronto Tri-Hospital Gestational Diabetes Project. Am J Obstet Gynecol. 1995; 173(1):146-156; doi: 10.1016/0002-9378(95)90183-3.

7. McFarland MB, Trylovich CG, Langer O. Anthropometric differences in macrosomic infants of diabetic and nondiabetic mothers. J Matern Fetal Med. 1998;7(6):292295; doi: 10.1002/(SICI)1520-6661(199811/12)7:6<292:: AID-MFM7>3.0.CO;2-A.

8. Miller JM Jr. Maternal and neonatal morbidity and mortality in cesarean section. Obstet Gynecol Clin North Am. 1988;15(4):629-638.

9. Casey BM, Lucas MJ, McIntire DD, Leveno KJ. Pregnancy outcomes in women with gestational diabetes compared with the general obstetric population. Obstet Gynecol. 1997;90(6):869-873; doi: 10.1016/S0029-7844(97)00 542-5.

10. Dang K, Homko C, Reece EA. Factors associated with fetal macrosomia in offspring of gestational diabetic women. J Matern Fetal Med. 2000;9(2):114-117; doi: 10.1002/(SICI)1520-6661(200003/04)9:2<114::AIDMFM5>3.0.CO;2-R.

11. Langer O, Levy J, Brustman L, Anyaegbunam A, Merkatz R, Divon M. Glycemic control in gestational diabe- 
tes mellitus - how tight is tight enough: small for gestational age versus large for gestational age? Am J Obstet Gynecol. 1989;161(3):646-653; doi: 10.1016/0002-9378 (89)90371-2.

12. Hollander MH, Paarlberg KM, Huisjes AJ. Gestational diabetes: a review of the current literature and guidelines. Obstet Gynecol Surv. 2007;62(2):125-136; doi: 10.1097/ 01.ogx.0000253303.92229.59.

13. Dyck R, Klomp H, Tan LK, Turnell RW, Boctor MA. A comparison of rates, risk factors, and outcomes of gestational diabetes between aboriginal and non-aboriginal women in the Saskatoon health district. Diabetes Care. 2002; 25(3):487-493; doi: 10.2337/diacare.25.3.487.

14. Hod M, Kapur A, Sacks DA, Hadar E, Agarwal M, Di Renzo GC, et al. The International Federation of Gynecology and Obstetrics (FIGO) initiative on gestational diabetes mellitus: a pragmatic guide for diagnosis, management, and care. Int J Gynaecol Obstet. 2015;131 Suppl 3:S173-S211; doi: 10.1016/S0020-7292(15)30007-2.

15. Barakat R, Pelaez M, Lopez C, Lucia A, Ruiz JR. Exercise during pregnancy and gestational diabetes-related adverse effects: a randomised controlled trial. $\mathrm{Br} \mathrm{J}$ Sports Med. 2013;47(10):630-636; doi: 10.1136/bjsports-2012091788.

16. Impact of physical activity during pregnancy and postpartum on chronic disease risk. Med Sci Sports Exerc. 2006;38(5):989-1006; doi: 10.1249/01.mss.0000218147. $51025.8 \mathrm{a}$

17. Cremona A, O'Gorman C, Cotter A, Saunders J, Donnelly $A$. Effect of exercise modality on markers of insulin sensitivity and blood glucose control in pregnancies complicated with gestational diabetes mellitus: a systematic review. Obes Sci Pract. 2018;4(5):455-467; doi: 10.1002/osp4.283.

18. Piantadosi S. Clinical trials: a methodologic perspective. Hoboken: Wiley; 2017.

19. Exercise during pregnancy and the postpartum period: number 267, January 2002 Committee on Obstetric Practice. Int J Gynecol Obstet. 2002;77(1):79-81; doi: 10.1016/ S0020-7292(02)80004-2.

20. Davies GA, Wolfe LA, Mottola MF, MacKinnon C. Joint SOGC/CSEP clinical practice guideline: exercise in pregnancy and the postpartum period. Can J Appl Physiol. 2003;28(3):330-341; doi: 10.1139/h03-024.

21. De Barros MC, Lopes MA, Francisco RP, Sapienza AD, Zugaib M. Resistance exercise and glycemic control in women with gestational diabetes mellitus. Am J Obstet Gynecol. 2010;203(6):556.e1-556.e6; doi: 10.1016/j. ajog.2010.07.015.

22. Committee Opinion No. 650 Summary: physical activity and exercise during pregnancy and the postpartum period. Obstet Gynecol. 2015;126(6):1326-1327; doi: 10.1097/ AOG.0000000000001209.

23. Share with Women. Exercise in pregnancy. J Midwifery Womens Health. 2014;59(4):473-474; doi: 10.1111/ jmwh.12218.

24. Casey BM, Mclntire DD, Leveno KJ. The continuing value of the Apgar score for the assessment of newborn infants. N Engl J Med. 2001;344(7):467-471; doi: 10.1056/ NEJM200102153440701.

25. Padayachee C, Coombes JS. Exercise guidelines for gestational diabetes mellitus. World J Diabetes. 2015;6(8): 1033-1044; doi: 10.4239/wjd.v6.i8.1033.

26. Crowther CA, Hiller JE, Moss JR, McPhee AJ, Jeffries WS, Robinson JS. Effect of treatment of gestational diabetes mellitus on pregnancy outcomes. N Engl J Med. 2005; 352(24):2477-2486; doi: 10.1056/NEJMoa042973.
27. Ju H, Rumbold AR, Willson KJ, Crowther CA. Borderline gestational diabetes mellitus and pregnancy outcomes. BMC Pregnancy Childbirth. 2008;8:31; doi: 10.1186/ 1471-2393-8-31.

28. Lawani M, Alihonou E, Akplogan B, Poumarat G. Exercise in pregnancy for elderly primigravida. $\mathrm{Br} \mathrm{J}$ Obstet Gynecol. 2008;51:476-480.

29. Juhl M, Olsen J, Andersen PK, Nøhr EA, Andersen AM. Physical exercise during pregnancy and fetal growth measures: a study within the Danish National Birth Cohort. Am J Obstet Gynecol. 2010;202(1):63.e1-63.e8; doi: 10.1016/j.ajog.2009.07.033.

30. Haakstad LA, Bø K. Exercise in pregnant women and birth weight: a randomized controlled trial. BMC Pregnancy Childbirth. 2011;11:66; doi: 10.1186/1471-239311-66.

31. Barakat R, Perales M, Bacchi M, Coteron J, Refoyo I. A program of exercise throughout pregnancy. Is it safe to mother and newborn? Am J Health Promot. 2014;29(1): 2-8; doi: 10.4278/ajhp.130131-QUAN-56.

32. Barakat R, Stirling JR, Lucia A. Does exercise training during pregnancy affect gestational age? A randomised controlled trial. Br J Sports Med. 2008;42(8):674-678; doi: 10.1136/bjsm.2008.047837.

33. The Apgar score. Pediatrics. 2015;136(4):819-822; doi: 10.1542/peds.2015-2651.

34. Ehrenstein V. Association of Apgar scores with death and neurologic disability. Clin Epidemiol. 2009;1:45-53; doi: 10.2147/CLEP.S4782. 